US WURK LXX (2021), p. 29

\title{
[1529] Gjin taalsoarch yn de soarch \\ De rol fan Frysk en Meartaligens yn de soarch- en wolwêzenssektor yn Fryslân
}

Myrthe Coret-Bergstra, Joana Duarte, Mirjam Günther-van der Meij \& Sibrecht Veenstra

\begin{abstract}
This article discusses how university-level healthcare and welfare course programs in the official bilingual Province of Friesland/Fryslân can best be aligned with healthcare practice regarding the use of Frisian and the inclusion of other forms of multilingualism. 15 interviews with healthcare and welfare professionals confirm previous findings on the significant role of minority languages in the healthcare and welfare sector. When minority languages, such as Frisian, are used the bond between healthcare recipient and care provider is strengthened and the patient is better able to communicate. However, the 9 interviews with lecturers, directors and team leaders of healthcare and welfare programs in Fryslân show that these pay little attention to Frisian and multilingualism in their curriculum. At the same time, it appears that those involved in the teaching understand the importance of addressing communication in Frisian and other languages and recognise that this aspect should be embedded in courses that focus on properly connecting with the personal context of the care recipient. These results will be discussed by providing recommendations for healthcare and welfare programs in higher education on how to incorporate minority languages, in particular, and multilingualism, in general.
\end{abstract}

\section{Ynlieding}

"In principe spreken we hier Nederlands, natuurlijk," jout in wurknimmer fan Veilig Thuis yn Ljouwert as antwurd op de fraach oft der in spesifyk belied is yn it oansprekken fan de kliïnten. Se seit fierder dat, as de kliïnt in oare taal praat, de wurknimmer him besiket oan te passen. Ut dit foarbyld docht bliken dat oare talen, ek regionale talen lykas it Frysk en syn fariëteiten, net altyd fanselssprekkend binne yn de soarch- en wolwêzenssektor. Dat byld wurdt befêstige yn de literatuer en blykt ek út de befiningen fan dit ûndersyk: hoewol't it kommunisearjen yn immen syn memmetaal hiel belangryk fûn wurdt yn de soarch- en wolwêzenssektor, hawwe net folle ynUs Wurk 70 (2021), s. 29-49; https://doi.org/10.21827/5fb7c8f6df202 


\section{US WURK LXX (2021), p. 30}

stellings dêr in offisjeel taalbelied foar. By de hbû-opliedingen Verpleegkunde, Vaktherapie en Social Work op de NHL Stenden Hogeschool yn Ljouwert is der op dit momint ek gjin eksplisyt omtinken foar minderheidstalen (regionale talen/dialekten en migrantetalen).

Minderheidstalen binne lykwols fan grut belang yn de soarch. Wagoner (2017) wiist der bygelyks op dat yn in twatalige regio lykas Wales kommunikaasje yn de memmetaal belangryk is foar de soarchfregers: yn de memmetaal is kommunikaasje makliker en kin better treast bean wurde (Wagoner, 2017). Yn de Nederlânske kontekst docht út ûndersyk ûnder sawol soarchferlieners as soarchfregers ek bliken dat it hiel belangryk fûn wurdt om yn de (regionale) memmetaal kommunisearje te kinnen (sjoch Afûk (2013) oer it Frysk, Loeven (2005) oer it Twintsk en Berends (2020) oer regionale talen yn hiel Nederlân).

Der liket dus in kleau te bestean tusken oan de iene kant de winsk fan soarchferlieners en soarchfregers om yn de memmetaal fan de pasjint te kommunisearjen, en oan de oare kant in tekoart oan taalbelied en omtinken foar regionale talen en meartaligens yn de soarch- en wolwêzensopliedingen. Om dy kleau oer te brêgjen, is it fan belang om te ûndersykjen hoe't it oanbod yn de soarch- en wolwêzensopliedingen it bêste oanslute kin by de praktyk en de winsken fan de soarchferlieners en soarchfregers.

Om in antwurd op dy fraach formulearje te kinnen, rjochtet dit ûndersyk him op it ferlet fan it wurkfjild yn de soarch- en wolwêzenssektor oangeande it brûken fan it Frysk (en oare minderheidstalen). Ek wurdt der ûndersyk dien nei karakteristike ûnderfinings yn relaasje ta it brûken fan it Frysk/meartaligens en nei it spesifike ferlet yn relaasje ta it Frysk (en oare minderheidstalen) fan de hbû-opliedingen oan NHL Stenden. Dêrby komt ek de fraach oan 'e oarder hoe't it ferlet fan 'e sektor en it oanbod fan 'e opliedings sa goed mooglik op elkoar oanslute kinne. It doel is om in antwurd te krijen op de folgjende ûndersyksfragen:

1) Wat is it ferlet fan it wurkfjild yn de soarch- en wolwêzenssektor oangeande it brûken fan it Frysk en oare minderheidstalen?

2) Wat binne spesifike ûnderfiningen en it spesifike ferlet yn relaasje ta it Frysk (en oare minderheidstalen) op de hbû-opliedingen oan NHL Stenden?

3) Hoe kin de Fryske taal yn it ramt fan de brede meartalige situaasje dy't de provinsje Fryslân typearret yn de hbû-soarchopliedingen yntegrearre wurde? 


\section{US WURK LXX (2021), p. 31}

\section{Taal yn de soarch- en wolwêzenssektor yn twatalige regio's}

Taal foarmet de kearn fan profesjonele sûnenssoarch. Dat is earder troch Clark (1983:807) sa beskreaun: "Without language, the work of a physician and that of a veterinarian would be nearly identical." Kommunikaasje yn de soarchsektor is al lang fan grut belang foar ûndersikers. Unfermogen om te kommunisearjen mei in soarchferliener behinderet net allinne de tagong ta sûnenssoarch, mar ûndergraaft ek it fertrouwen yn de kwaliteit fan medyske soarch (Johnson, Noble, Mattheus \& Aguilar, 1998). Der binne benammen soargen oer problemen dy't har foardwaan kinne as pasjinten en soarchferlieners net deselde earste taal prate (Robinson, 2002). Ferskillende ûndersikers (Anderson, Scrimshaw, Fulilove, Fielding \& Normand, 2003; Chen, Youdelman \& Brooks, 2007) sizze dat goede kommunikaasje mei pasjinten beskiedend wêze kin om emosjonele, etyske en juridyske redenen. Yn meartalige lannen, en foaral yn lannen of regio's dy't offisjeel mear as ien taal hawwe, kin kommunikaasje in útdaging wêze (Lavizzo-Mourey, 2007). Der binne mooglike stappen dy't nommen wurde kinne om sokke útdagingen oan te pakken. Schenker, Lo, Ettinger en Fernandez (2008) jouwe dêr in foarbyld fan. De ûndersikers suggerearje dat spesifike situaasjes dy't yn de twadde taal (T2) as in taaldrompel ûnderfûn wurde, effisjint oefene wurde moatte. Soarchferlieners soene yn dat ramt traind wurde moatte yn de T2 fan harren kliïnten.

De rol fan minderheidstalen yn soarch(opliedingen) is net fanselssprekkend: Wagoner (2017) wiist derop dat de needsaak fan twatalich ûnderwiis yn konteksten lykas ynstellingen foar sûnenssoarch net algemien erkend wurdt. Yn Wales bygelyks libbet it idee, sa't Davies (2009) it seit, dat "se dochs allegear Ingelsk kinne", en dat soarchpersoniel dêrom gjin Welsh hoecht te praten. Nettsjinsteande de alles omfetsjende ientalige ideologyen is der hieltyd mear ûndersyk dêr't út bliken docht dat der ferlet is fan meartalich soarchpersoniel. Wagoner (2017) hellet twa haadredenen nei foaren: (1) pasjinten dy't net by steat binne om har te uterjen en oaren te begripen mei deselde feardigens yn har twadde taal as yn har earste taal; en (2) de pasjinten wat treast jaan yn in tiid dat se it dreech hawwe en/of kwetsber binne troch se oan te sprekken yn de earste taal.

Fierder befêstigje Roberts en Paden (2000:75) dat der ferlet is fan twatalich personiel; se binne fan miening dat "(...) circumstances of stress and vulnerability, denying opportunities for clients to communicate in their preferred language may place them [multilingual patients] at a personal disadvantage and compromise their health chances". Guon meartalige regio's yn Europa binne ta it ynsjoch kommen dat der ferlet is fan twatalige 


\section{US WURK LXX (2021), p. 32}

sûnenssoarch en hawwe dêr oan wurke. Yn oerienstimming mei de oanrikkemandaasjes fan de Welshe taalkommissaris jout it soarchpersoniel út Wales yn in enkête oan (Irvine et al., 2006), dat de mooglikheid om it Welsh te brûken derfoar soarge hat dat âldere pasjinten dy't Welsh prate harren nofliker en komfortabeler fielden.

Ek yn Noard-Dútslân is der ferlet fan soarchpersoniel dat de regionale taal prate kin. Ut ûndersyk fan de Berufsbildende Schule (BBS, beropsûnderwiis) Wildeshausen yn Noard-Dútslân die bliken dat $75 \%$ fan de ûnderfrege soarchfregers yn harren jeugd Platdútsk (de regionale taal) sprutsen (Schlarmann, 2016). Sy joegen oan dit noch altyd graach te sprekken en $80 \%$ joech eksplisyt oan mei soarchferlieners it leafst Platdútsk prate te wollen (Schlarmann, 2016). Op BBS Wildeshausen wurde der inisjativen ûntwikkele om oan dizze fraach foldwaan te kinnen. Sa is der in brosjuere "Plattdeutsch in der Pflege" útbrocht (Berufsbildende Schulen des Landkreises Oldenburg, 2015), wêryn't in wurdlist yn it Platdútsk te finen is, spesjaal rjochte op de âldereinsoarch en de ferpleechsoarch. "Die Erstsprache der zu Pflegenden ist ein Türöffner, eine Brücke, und vereinfacht oftmals notwendige Handlungen in der Pflege", stiet yn it foaropwurd (Berufsbildende Schulen des Landkreises Oldenburg, 2015:2). Dêryn wurdt oanjûn dat it brûken fan de earste taal fan de soarchfreger de kommunikaasje makliker makket en boppedat in brêge slacht tusken soarchferliener en soarchfreger. Op de BBS Wildeshausen hawwe studinten dêrneist de mooglikheid om lessen taalfeardigens Platdútsk te folgjen (Schlarmann, 2016). Ek op guon oare ûnderwiisynstellings, lykas de Katholieke Akademie Stapelfeld wurdt "Platt in der Pflege" ûnderwiisd (Institut für niederdeutsche Sprache, 2016).

Yn Fryslân spilet dy kwestje ek. De Afûk ferrjochte yn 2013 in ûndersyk nei de rol fan it Frysk yn de soarch. Yn dat ûndersyk waard sawol soarchpersoniel as pasjinten frege nei harren ûnderfiningen mei en mieningen oer de rol fan it Frysk yn it deistich libben. Mear as de helte fan de 395 ûnderfrege pasjinten hat it Frysk as memmetaal. Foaral âldere respondinten joegen oan dat it praten fan de (minderheids-)memmetaal natuerliker oanfielde, dat it harren in gefoel fan feilichheid joech en dat se harren dêr mear thús by fielden. In grutte mearderheid $(92 \%)$ fan de respondinten ferklearre in dúdlike foarkar te hawwen foar konversaasje yn de eigen memmetaal. Troch yn de memmetaal te kommunisearjen fielden se har mear op harren gemak en wiene se better by steat om oan te jaan hoe't se har fielden. Boppedat joegen de measte soarchfregers oan dat se yn de hjoeddeistige soarchfoarsjenning geregeld harren memmetaal prate koene en dat se dêr tefreden oer wiene. Fan it soarchpersoniel koe in mearderheid fan de wurknimmers 


\section{US WURK LXX (2021), p. 33}

Frysk prate. $90 \%$ fan it personiel besocht safolle mooglik de memmetaal fan de soarchfreger te praten. De soarchferlieners ferklearren dat se troch Frysk te praten de soarchfreger optimaal soarch jaan koene. Se joegen ûnder mear oan dat Frysk praten:

- de ferbining tusken soarchpersoniel en soarchfreger fersterke;

- derfoar soarge dat soarchfregers har uterje koene sûnder dat se om wurden sykje moasten;

- it de soarchfregers makliker makke om lestige saken te bepraten.

Respondinten fan beide enkêtes wiisden fierder op it belang fan de minderheidstaal by it erkennen fan de soarchfreger as in kompleet persoan, rekkening hâldend mei syn of har taal en identiteit. In ferpleechster yn Wales ferklearre: "Soms betsjut it datst, troch harren eigen taal te praten - harren eigen taal, snapste - se as in folslein persoan sjochst "(Irvine et al., 2006).

Der binne ferskillende oare ûndersiken dy't it brûken fan minderheidstalen yn de soarch yn Nederlân analysearren. Sa waard bygelyks it brûken fan regionale talen yn de soarch yn de Achterhoek ûndersocht troch Krosenbrink (1983). Ferpleechkundigen en oare meiwurkers fan ferpleechhuzen en sikehuzen waarden yn it ramt fan dat ûndersyk ynterviewd oer harren taalgebrûk. Ut de resultaten die bliken dat $73 \%$ fan de meiwurkers it Achterhoeksk brûkte en neffens $71 \%$ fan de meiwurkers waard it brûken fan regionale taal yn soarchynstellingen tolerearre. Ut de resultaten die bliken dat ek de measte âlderen in regionale taal praten $(75 \%)$.

De ferfolchstúdzje oer it brûken fan regionale talen yn de soarch yn de Achterhoek waard útfierd troch Loeven (2005). Soarchferlieners en soarchfregers yn twa sikehuzen (ien yn de Achterhoek en ien yn Twinte) waard frege om in fragelist yn te foljen mei iepen en sletten fragen, om sa mear ynsjoch te krijen yn it gebrûk fan regionale talen en de foar- en neidielen dêrfan yn sikehuzen. Sawol soarchfregers as soarchferlieners rapportearren dat it brûken fan regionale talen yn sikehuzen foardielen joech. Sa joegen pasjinten oan dat se de regionale taal foaral brûkten as se mei ferpleechsters praten. Yn petearen mei dokters waard de regionale taal hast noait brûkt. Dat soe ynterpretearre wurde kinne as it resultaat fan iepentlik prestiizje. Prestiizje beskriuwt it nivo fan respekt dat takend wurdt oan in dialekt of taal, ferlike mei oare talen of dialekten yn in mienskip (Labov, 1972). It brûken fan Twintsk of Achterhoeksk waard troch sawol soarchferlieners as pasjinten posityf wurdearre. Fan beide kanten waard as foardiel neamd dat it de kommunikaasje makliker makke. Dêrnei kamen foardielen as it kreëarjen fan in goede sfear (neffens de soarchfregers) en it krijen fan in fertroud 


\section{US WURK LXX (2021), p. 34}

gefoel (neffens de soarchferlieners). Dat it brûken fan regionale taal yn de soarch noch hieltyd spilet ûnder soarchferlieners is dúdlik: yn desimber 2019 die de redaksje fan it blêd Nursing in oprop op Facebook mei de fraach oft de lêzers wol ris yn in regionale taal of dialekt mei harren kliïnten of pasjinten praten. Ut de 275 reaksjes die bliken dat in soad dat diene en dat it brûken fan in regionale taal faak beskôge waard as in mearwearde yn it kontakt tusken soarchferlieners en soarchfregers (Berends, 2020).

Om soarch yn de memmetaal oanbiede te kinnen, is it fan belang om meartalich soarchpersoniel te hawwen. Nettsjinsteande it feit dat guon derfoar pleitsje om tolken yn te skeakeljen, hâlde in soad taaldeskundigen út dat tolken net foldogge oan it ferlet fan de soarchfreger en dat it ynskeakeljen fan in tolk net itselde is as it wurkjen mei twatalich personiel. Krekt oarsom, Coffi (2005) suggerearret dat it de bêste oplossing is om de 'tuskenpersoan' te omsilen, omdat dokters harren pasjint in heger soarchnivo biede kinne en in heger nivo fan autoriteit, feardichheden en/of fertrouwen mei foar de soarchfreger oer hawwe kinne as it kontakt streekrjocht is. Wol wiist Coffi (2005) derop dat it ek in negative kant hat as men net gebrûk makket fan in tolk: dat kin liede ta útstel fan de kommunikaasje, omdat der wachte wurde moat op in twatalich personielslid yn de folgjende ploech (by ploegetjinst). Wagoner (2017) docht dêrom de suggestje om de taalfeardigens fan soarchpersoniel te fergrutsjen, sadat soarchfregers yn harren foarkarstaal behannele wurde kinne, sûnder help fan tolken en sûnder it ûntstean fan wachttiden. Dêrom soene migranten dy't op it stuit wurkje of wurk sykje yn de sûnenssoarch yn twatalige regio's, mear stipe wurde moatte om net ien, mar (op syn minst) twa talen fan de gastmienskip te learen, om sa oan it ferlet fan soarchfregers te foldwaan. Mar ek oaren foar wa't de regionale taal net de memmetaal is, soene oan harren taalfeardichheden wurkje kinne om basiskommunikaasje yn de regionale taal te garandearjen.

It taalgebrûk yn ferpleechhuzen yn Fryslân is ûndersocht yn opdracht fan de provinsje Fryslân (Blömer, Boneschansker, \& van der Tuin, 2001). Ut de resultaten fan dat ûndersyk die bliken dat bewenners it brûken fan it Frysk troch ferpleechkundigen en oare personielsleden yn ferpleechhuzen yn Fryslân posityf wurdearren. Hoewol't der gjin sprake is fan konkreet Frysk taalbelied yn de sûnenssoarch, ek net yn ferpleechhuzen, blykt út earder ûndersyk (Afûk, 2013) dat it brûken fan it Frysk troch helpferlieners tige algemien is, offhinklik fan de fraach hoefolle Frysk oft der yn it oanbelangjende gebiet praat wurdt. Dêrom ropt it ûndersyksprojekt oer meartaligens yn Fryske soarchsituaasjes (Afûk, 2013) op ta taalbelied, om de hjoeddeistige praktyk te strukturearjen en it taalbewustwêzen by it soarchpersoniel te 


\section{US WURK LXX (2021), p. 35}

fergrutsjen. Der is lykwols gjin ûndersyk bekend dat de rol fan Frysk en meartaligens yn de soarch- en wolwêzensopliedingen yn it HBÛ bestudearret. Troch alfêst te ynvestearjen yn de oplieding fan de jonge soarchferlieners komt der mear ynsjoch yn it belang fan meartaligens yn de kommunikaasje mei pasjinten en kliïnten. Boppedat kin tefoarenkommen wurde dat der in tekoart ûntstiet oan personiel dat taalfeardich is yn it Frysk.

In mooglike rûte dy't liede kin ta it fergrutsjen fan taalbewustwêzen by soarchprofessionals rint dus fia harren opliedingstrajekt. It doel fan it hjir útfierde ûndersyk is dan ek om it ferlet fan de soarchsektor oangeande it brûken fan it Frysk en oare talen te ferbinen mei de wurkwizen en it ferlet by de oplieding fan soarchmeiwurkers yn it hbû (oplieding Vaktherapie en Verpleegkunde, NHL Stenden Hogeschool). It ûndersyk boude ek fierder op boppesteande ynsichten troch it ûndersyksgebiet te fergrutsjen: neist it ûndersyk yn de soarchferliening waard ek ûndersocht wat der bart yn de wolwêzenssektor (oanslutend by de oplieding Social Work, NHL Stenden Hogeschool). Dêrneist waard troch de wei fan ynterviews frege nei persoanlike ûnderfiningen om in sa konkreet mooglik byld te krijen fan de meartalige situaasjes dy't har foardogge yn de soarch- en wolwêzenssektor. It úteinlike doel is ommers om dy befiningen om te setten yn konkrete oanrekommandaasjes foar de opliedingen en konkrete ideeën foar it ûntwikkeljen fan lesmateriaal foar boppeneamde opliedingen.

\section{Metoade}

\subsection{Semystrukturearre ynterviews}

Om de ûndersyksfragen te beäntwurdzjen, is keazen foar in kwalitatyf ûndersyksdesign, om sa ynformaasje te krijen dy't in oanfolling is op it mear kwantitative Afûk-ûndersyk fan 2013, dat basearre is op in enkête. Der binne 24 semystrukturearre ynterviews (Hopf, 2004) offnommen, dat wol sizze: ynterviews wêrby't iepen fragen steld waarden, mar wêrby't de eksakte formulearring fan dy fragen en de folchoarder wêryn't se steld waarden, ôfhinklik wiene fan it petear. Fan de 24 ynterviews waarden der njoggen ôfnommen oan belutsenen fan de hbû-oplieding en 15 oan wurknimmers út de soarch- en wolwêzenssektor. De ynterviews waarden meastentiids telefoanysk of digitaal ôfnommen, want dit part fan it ûndersyk krige allegear syn beslach yn 'e tiid fan de COVID-19-krisis. Der binne 4 ferskillende sets ynterviewgidsen foar dit doel opsteld: 1) foar soarch- en wolwêzensmeiwurkers, 2) foar managers fan soarch- en wolwêzensynstellingen, 3) foar dosinten en 4) foar teamlieders/direkteuren. Dêrby binne de fragen oanpast 


\section{US WURK LXX (2021), p. 36}

oan de ferskillende rollen fan de partisipanten yn kwestje. Alle ynterviews bestiene út (fragen by) de folgjende ûnderdielen:

- fyzje en belied op it mêd fan meartaligens;

- belang fan it Frysk (en oare talen) yn de soarch-/wolwêzenssektor;

- bewustwêzen fan meartaligens yn it algemien;

- persoanlike ûnderfiningen mei meartalige situaasjes.

Dêrneist is der nei inkele eftergrûnaspekten frege, lykas funksje, leeftyd, memmetaal en oare fiertalen. De ynterviews binne opnommen en dêrnei verbatim transkribearre.

\subsection{Stekproef}

Yn totaal binne der 24 dielnimmers ûnderfrege. Dêrby waard gebrûk makke fan teoretyske of snowball sampling (Mack et al., 2005), dat wol sizze dat der doelbewust kontakt socht is mei persoanen dy't mear, oare of spesifike ynformaasje jaan koene oer it ûnderwerp. Sa'n kontaktpersoan waard frege oft hy/sy noch oaren wist dy't oan de stekproef meidwaan wolle soene. Sa koe soarge wurde foar mear dielnimmers fan ien oplieding/ynstelling. In oersjoch fan de 15 dielnimmers út it wurkfjild stiet hjirûnder yn tabel 1. Opfallend is dat 13 fan de 15 dielnimmers Frysk prate, en noch 1 dielnimmer oanjout it wol te ferstean. Foar 9 dielnimmers is Frysk de memmetaal. De dielnimmers wurkje by 6 ferskillende ynstellingen: trije sikehûzen (MCL, Sionsberg en Nij Smellinghe), de GGZ (psychische hulpverlening), Veilig Thuis (advys en stipe húslik geweld en bernemishanneling) en Zienn (opfang en stipe foar dakleazen).

Tabel 1: Oersjoch ynterviews wurkfjild

\begin{tabular}{|c|c|c|c|c|c|c|c|c|c|}
\hline $\mathrm{Nr}$ & $\begin{array}{l}\text { Ge- } \\
\text { slacht }\end{array}$ & $\begin{array}{l}\text { Digi- } \\
\text { taal } \\
\text { yn- } \\
\text { ter- } \\
\text { view }\end{array}$ & $\begin{array}{l}\text { Lingte } \\
\text { Inter- } \\
\text { view } \\
\text { (min) }\end{array}$ & $\begin{array}{l}\text { Mem- } \\
\text { me- } \\
\text { taal }\end{array}$ & $\begin{array}{l}\text { Praat } \\
\text { Frysk }\end{array}$ & Funksje & Bedriuw & Type & $\begin{array}{l}\text { Slút meast } \\
\text { oan by }\end{array}$ \\
\hline 1 & $\mathrm{~F}$ & $\mathrm{Ja}$ & 21 & FR & $\mathrm{Ja}$ & $\begin{array}{l}\text { Team- } \\
\text { lieder }\end{array}$ & MCL & $\begin{array}{l}\text { Sike- } \\
\text { hûs }\end{array}$ & $\begin{array}{l}\text { Ferpleech- } \\
\text { kunde }\end{array}$ \\
\hline 2 & $\mathrm{~F}$ & $\mathrm{Ja}$ & 19 & NL & $\mathrm{Ja}$ & $\begin{array}{l}\text { Koör- } \\
\text { di- } \\
\text { nator }\end{array}$ & Zienn & $\begin{array}{l}\text { Op- } \\
\text { fang/ } \\
\text { help }\end{array}$ & Social work \\
\hline 3 & $\mathrm{~F}$ & $\mathrm{Ja}$ & 20 & FR & $\mathrm{Ja}$ & $\begin{array}{l}\text { Team- } \\
\text { lieder }\end{array}$ & MCL & Sikehûs & $\begin{array}{l}\text { Ferpleech- } \\
\text { kunde }\end{array}$ \\
\hline 4 & $\mathrm{~F}$ & $\mathrm{Ja}$ & 30 & FR & $\mathrm{Ja}$ & $\begin{array}{l}\text { Ma- } \\
\text { nager }\end{array}$ & $\begin{array}{l}\text { Sions- } \\
\text { berg }\end{array}$ & $\begin{array}{l}\text { Me- } \\
\text { dyske } \\
\text { klinyk }\end{array}$ & $\begin{array}{l}\text { Ferpleech- } \\
\text { kunde }\end{array}$ \\
\hline
\end{tabular}


US WURK LXX (2021), p. 37

\begin{tabular}{|c|c|c|c|c|c|c|c|c|c|}
\hline 5 & $\mathrm{~F}$ & $\mathrm{Ja}$ & 17 & FR & $\mathrm{Ja}$ & $\begin{array}{l}\text { Ma- } \\
\text { nager }\end{array}$ & GGZ & $\begin{array}{l}\text { Psy- } \\
\text { chyske } \\
\text { help }\end{array}$ & Fakterapy \\
\hline 6 & M & Nee & 19 & NL & $\mathrm{Ja}$ & $\begin{array}{l}\text { Fer- } \\
\text { pleech- } \\
\text { kun- } \\
\text { dige }\end{array}$ & MCL & $\begin{array}{l}\text { Sike- } \\
\text { hûs }\end{array}$ & $\begin{array}{l}\text { Ferpleech- } \\
\text { kunde }\end{array}$ \\
\hline 7 & F & $\mathrm{Ja}$ & 18 & NL & $\mathrm{Ja}$ & $\begin{array}{l}\text { Maat- } \\
\text { skiplik } \\
\text { wurker }\end{array}$ & $\begin{array}{l}\text { Vei- } \\
\text { lig } \\
\text { Thuis }\end{array}$ & $\begin{array}{l}\text { Op- } \\
\text { fang/ } \\
\text { help }\end{array}$ & Social work \\
\hline 8 & $\mathrm{~F}$ & $\mathrm{Ja}$ & 20 & FR & $\mathrm{Ja}$ & $\begin{array}{l}\text { Maat- } \\
\text { skiplik } \\
\text { wurker }\end{array}$ & $\begin{array}{l}\text { Vei- } \\
\text { lig } \\
\text { Thuis }\end{array}$ & $\begin{array}{l}\text { Op- } \\
\text { fang/ } \\
\text { help }\end{array}$ & Social work \\
\hline 9 & $\mathrm{~F}$ & $\mathrm{Ja}$ & 10 & $\begin{array}{l}\text { Ara- } \\
\text { bysk* }\end{array}$ & $\mathrm{Ja}$ & $\begin{array}{l}\text { Sta- } \\
\text { zjêre } \\
\text { fer- } \\
\text { pleech- } \\
\text { kunde }\end{array}$ & MCL & $\begin{array}{l}\text { Sike- } \\
\text { hûs }\end{array}$ & $\begin{array}{l}\text { Fer- } \\
\text { pleechkunde }\end{array}$ \\
\hline 10 & $\mathrm{~F}$ & $\mathrm{Ja}$ & 18 & FR & $\mathrm{Ja}$ & $\begin{array}{l}\text { Bege- } \\
\text { lieder }\end{array}$ & Zienn & $\begin{array}{l}\text { Op- } \\
\text { fang/ } \\
\text { help }\end{array}$ & Social work \\
\hline 11 & $\mathrm{~F}$ & $\mathrm{Ja}$ & 17 & FR & $\mathrm{Ja}$ & $\begin{array}{l}\text { Tera- } \\
\text { peut }\end{array}$ & GGZ & $\begin{array}{l}\text { Psy- } \\
\text { chys- } \\
\text { ke help }\end{array}$ & $\begin{array}{l}\text { Fak- } \\
\text { terapy }\end{array}$ \\
\hline 12 & $\mathrm{~F}$ & $\mathrm{Ja}$ & 16 & NL & $\begin{array}{l}\text { Allin- } \\
\text { ne } \\
\text { fer- } \\
\text { stean }\end{array}$ & $\begin{array}{l}\text { Tera- } \\
\text { peut }\end{array}$ & GGZ & $\begin{array}{l}\text { Psy- } \\
\text { chys- } \\
\text { ke help }\end{array}$ & $\begin{array}{l}\text { Fak- } \\
\text { terapy }\end{array}$ \\
\hline 13 & $\mathrm{~F}$ & $\mathrm{Ja}$ & 18 & FR & $\mathrm{Ja}$ & $\begin{array}{l}\text { Tera- } \\
\text { peut }\end{array}$ & GGZ & $\begin{array}{l}\text { Psy- } \\
\text { chys- } \\
\text { ke help }\end{array}$ & $\begin{array}{l}\text { Fak- } \\
\text { terapy }\end{array}$ \\
\hline 14 & M & $\mathrm{Ja}$ & 22 & NL & Nee & $\begin{array}{l}\text { Tera- } \\
\text { peut }\end{array}$ & GGZ & $\begin{array}{l}\text { Psy- } \\
\text { chys- } \\
\text { ke help }\end{array}$ & $\begin{array}{l}\text { Fak- } \\
\text { terapy }\end{array}$ \\
\hline 15 & $\mathrm{~F}$ & $\mathrm{Ja}$ & 14 & FR & $\mathrm{Ja}$ & $\begin{array}{l}\text { Fer- } \\
\text { pleech- } \\
\text { kun- } \\
\text { dige }\end{array}$ & $\begin{array}{l}\text { Nij } \\
\text { Smel- } \\
\text { lin- } \\
\text { ghe }\end{array}$ & $\begin{array}{l}\text { Sike- } \\
\text { hûs }\end{array}$ & $\begin{array}{l}\text { Fer- } \\
\text { pleechkunde }\end{array}$ \\
\hline
\end{tabular}

* Wierskynlik Arabysk, mar fanwege privacyregelingen kin dat net befêstige wurde

De dielnimmers fan de opliedingen by NHL Stenden wurde hjirûnder werjûn yn tabel 2. Foar 3 fan harren is Frysk de memmetaal, wylst yn totaal 6 dielnimmers oanjouwe Frysk te praten. De oare 3 jouwe oan wol Frysk te ferstean. 


\section{US WURK LXX (2021), p. 38}

Tabel 2: Oersjoch ynterviews yn de opliedingen fan NHL Stenden

\begin{tabular}{|c|c|c|c|c|c|c|c|}
\hline Nr. & $\begin{array}{l}\text { Ge- } \\
\text { slacht }\end{array}$ & $\begin{array}{l}\text { Digi } \\
\text { taal } \\
\text { ynter- } \\
\text { view }\end{array}$ & $\begin{array}{l}\text { Lingte } \\
\text { Ynter- } \\
\text { view } \\
\text { (min) }\end{array}$ & $\begin{array}{l}\text { Mem- } \\
\text { me- } \\
\text { taal }\end{array}$ & $\begin{array}{l}\text { Praat } \\
\text { Frysk }\end{array}$ & Funksje & Oplieding \\
\hline 16 & $\mathrm{~F}$ & $\mathrm{Ja}$ & 11 & NL & $\mathrm{Ja}$ & $\begin{array}{l}\text { Opliedings- } \\
\text { direkteur }\end{array}$ & Social work \\
\hline 17 & $\mathrm{~F}$ & $\mathrm{Ja}$ & 14 & FR & $\mathrm{Ja}$ & $\begin{array}{l}\text { Opliedings- } \\
\text { direkteur }\end{array}$ & $\begin{array}{l}\text { Fakterapy \& } \\
\text { Ferpleechkunde }\end{array}$ \\
\hline 18 & $\mathrm{~F}$ & Nee & 24 & FR & $\mathrm{Ja}$ & Teamlieder & Fakterapy \\
\hline 19 & M & $\mathrm{Ja}$ & 13 & NL & $\mathrm{Ja}$ & Teamlieder & Ferpleechkunde \\
\hline 20 & $\mathrm{~F}$ & $\mathrm{Ja}$ & 19 & NL & $\mathrm{Ja}$ & Teamlieder & Social work \\
\hline 21 & $\mathrm{~F}$ & $\mathrm{Ja}$ & 14 & NL & $\begin{array}{l}\text { Allinne } \\
\text { ferstean }\end{array}$ & Dosint & Fakterapy \\
\hline 22 & $\mathrm{~F}$ & Nee & 16 & NL & $\begin{array}{l}\text { Allinne } \\
\text { ferstean }\end{array}$ & Dosint & Fakterapy \\
\hline 23 & M & $\mathrm{Ja}$ & 15 & FR & $\mathrm{Ja}$ & Dosint & Social work \\
\hline 24 & M & $\mathrm{Ja}$ & 17 & NL & $\begin{array}{l}\text { Allinne } \\
\text { ferstean }\end{array}$ & Dosint & Ferpleechkunde \\
\hline
\end{tabular}

Yn totaal giet it om data basearre op ynterviews mei 19 froulju en 5 manlju; foar 12 is Frysk de memmetaal. De ynterviews hawwe yn totaal in lingte fan 422 minuten, dus 7 oeren en 2 minuten.

\subsection{Analyze}

Foar de analyze fan de ynterviews is gebrûk makke fan in thematic analysis (Braun \& Clarke, 2006), wêrby't ynduktive en deduktive wurkwizen kombinearre waarden om de rûge data en datastjoerde koades ynterpretearje te kinnen. Omdat de ynterviews net folslein strukturearre wiene en guon ûnderwerpen op ferskillende plakken yn de ynterviews oan bod komme, koene de antwurden net simpelwei per fraach analysearre wurde. Om de data per tema organisearje te kinnen wurdt de analyze útfierd neffens de seis analyzefazen lykas beskreaun troch Braun en Clarke (2006:87). ${ }^{1}$ Dêr kamen 4 haadtema's mei inkele subtema's út nei foaren, dy't hjirûnder werjûn wurde yn tabel 3. Boppedat is oanjûn hokker persintaazje fan de data oft yn it ramt fan dizze tema's kodearre is. Ferskate ekstrakten binne lykwols foar mear as ien tema kodearre.

1. De 6 fazen fan Braun \& Clarke (2006) binne: 1) Familiarisaasje mei de data, 2) foarriedige koades kreëarje, 3) tema's sykje, 4) tema's beoardiele, 5) definitive tema's bepale, 6) rapport skriuwe. Troch it trochrinnen fan dizze stappen kin út kwalitative data stapsgewiis de wichtichste ynformaasje helle wurde. 


\section{US WURK LXX (2021), p. 39}

Tabel 3: Definitive tema's n.o.f. de thematic analysis

\begin{tabular}{|c|c|}
\hline & $\begin{array}{l}\text { \% fan kodearrings yn de data } \\
\text { (Totaal: } 472 \text { kodearre ekstrakten) }\end{array}$ \\
\hline 1. Belang fan talen & - \\
\hline a. Belang fan it Frysk & $18 \%$ \\
\hline i. Praten fs. Ferstean & $3 \%$ \\
\hline b. Belang fan it Ingelsk & $6 \%$ \\
\hline c. Belang fan oare talen & $8 \%$ \\
\hline d. Fertalen \& ynset fan tolken & $8 \%$ \\
\hline 2. $\quad$ Ferskaat & - \\
\hline a. $\quad$ Oansluting by de soarchfreger & $10 \%$ \\
\hline b. $\quad$ Kultuerferskillen & $7 \%$ \\
\hline $\begin{array}{l}\text { c. } \begin{array}{l}\text { Frysktaligen fs. Net-Frysktaligen yn de } \\
\text { oplieding }\end{array}\end{array}$ & $4 \%$ \\
\hline d. $\quad$ Bewustwêzen fan meartaligens & $3 \%$ \\
\hline 3. Wearde memmetaal & $2 \%$ \\
\hline a. Skeppen fan in bân & $5 \%$ \\
\hline b. Uterjen fan gefoelens & $6 \%$ \\
\hline 4. Taalbelied & $21 \%$ \\
\hline
\end{tabular}

Yn de resultateseksje binne foar elk tema de belangrykste befiningen beskreaun.

\section{Resultaten}

\subsection{Tema 1: It belang fan talen}

It belang fan it Frysk

Alle ynterviewde persoanen, sawol Frysktalich as net-Frysktalich, joegen oan wol it belang te sjen fan it Frysk yn de soarch- en wolwêzenssektor yn Fryslân; hoe grut oft dat belang is, dêr ferskilden de mieningen oer. Partisipant 11 (P11), fakterapeut by GGZ, seit: "Ik vind het héél héél erg belangrijk.", mar P10, begelieder by Zienn, neamt it "Mooi meegenomen, maar niet echt belangrijk" om Frysk te praten as soarchferliener. Foar in soad meiwurkers yn de soarch seit it himsels dat guon soarchfregers Frysk 


\section{US WURK LXX (2021), p. 40}

prate en dat sy dêr as soarchferliener yn fasilitearje moatte. Dat men jin yn de memmetaal uterje kin en mei, wurdt faak belangryk neamd.

P5, manager fan in GGZ-ôfdieling, seit dêr bygelyks oer:

"Ik vind het belangrijk dat je in je eigen moedertaal kunt spreken en verstaan wordt en begrepen wordt."

P7, meiwurker by Veilig thuis, seit:

"Dat is denk ik in heel veel takken van sport binnen de hulpverlening wel zo, dat het meerwaarde heeft als mensen hun verhaal in het Fries kunnen doen."

By de opliedingen wurdt it belang fan it Frysk foar it wurkfjild wol sjoen. Dat wol sizze, it is bekend dat yn it wurkfjild in soad Frysk praat wurdt troch soarchfregers en it wurdt ek belangryk fûn dat dat sa bliuwe kin.

P23, dosint Social Work, seit:

"Nou vooral als je hier in de regio actief bent. Ik vind het aansluiten bij de regio heel erg belangrijk, dat je in ieder geval het Fries kunt verstaan, maar ook gevoeligheid voor taal en identiteit ontwikkelt, als je hier in deze regio actief bent als sociaal werker."

Om de studinten eksplisyt Frysk oan te learen, liket lykwols gjinien in goed idee ta; dat soe mar foar in lytse groep studinten relevant wêze en der soe net folle animo foar bestean, lykas hjirûnder ek te lêzen is by it tema "Frysktaligen fs. net-Frysktaligen yn de opliedingen".

Prate fs. ferstean

In soad meiwurkers jouwe oan dat se it Frysk prate kinne of op syn minst ferstean, en dat se it oars wol stadichwei leare yn harren wurk. Oer it algemien wurdt hieltyd nei foaren brocht dat Frysk ferstean belangryk is en Frysk prate minder, mar dat it wol as mearwearde sjoen wurdt.

"Ik weet dat de Fries het al op prijs stelt als je haar of hem kunt verstaan. Dat wordt al gewaardeerd want dan kunnen zij in ieder geval in hun taal blijven spreken, dus dat is al belangrijk. Kun je het dan ook nog terugspreken dan is dat natuurlijk alleen nog maar een meerwaarde," seit P19, teamlieder oplieding Ferpleechkunde.

Ek yn it MCL wurdt foaral belang hechte oan de passive behearsking fan it Frysk: "Ik vind wel als je als medewerker hier werkt dat het heel fijn is dat je de Friese taal in ieder geval verstaat en dat je het begrijpt." (P3, unitlieder 


\section{US WURK LXX (2021), p. 41}

MCL). In ferpleechkundige út Nij Smellinghe is it dêrmei iens: "Ik denk niet eens dat je het zelf hoeft te spreken, maar dat je het een beetje kunt verstaan wel, dat vind ik wel belangrijk." (P15).

$\mathrm{Al}$ mei al is it belang fan it Frysk ferstean dus grut, foarmet praten in mearwearde, mar sjogge de measte belutsenen by de opliedingen it eksplisyt oanbieden fan it Frysk dochs net as in opsje, omdat soks mar foar in pear studinten oantreklik wêze soe.

\section{It belang fan it Ingelsk, oare talen en oersetten}

Ingelsk spilet in rol yn de oplieding (bygelyks yn fakliteratuer) en soms ek dêrbûten, omdat it al gau de Lingua Franca is as men beide net deselde taal praat. Der wurdt ek oanjûn dat it brûken fan it Ingelsk wol hiel bot fan de doelgroep offhinget en dat by it grutste diel fan de soarchfregers dochs benammen Nederlânsk en Frysk in rol spylje yn Fryslân. Wat bygelyks migrantetalen oanbelanget, dêrfoar waard oanjûn dat men dy talen net op sa'n grutte skaal tsjinkomt dat der wat mei dien wurde moat. P9, dy't sels Arabysk praat, jout oan dat se mar twa kear Arabysk mei pasjinten praat hat. As oare talen har yn it wurkfjild foardogge, wurde der meastal ad hoc oplossingen betocht om der mei om te gean. Soms wurdt it Ingelsk as Lingua Franca brûkt, soms is oersetten nedich. Faak wurdt dan earst yntern socht nei immen dy't de taal wol machtich is en eventueel wurdt gebrûk makke fan in tolketelefoan. Dat wurdt sjoen as in needsaaklik rêdingsmiddel, omdat men net sels alle talen prate kin. "Die tolkentelefoon kan een gesprek van mens tot mens nooit vervangen," jout P2 oan. "Maar stel je voor dat ik een opleiding Tigrinya zou doen, dan ben ik pas over 10 jaar op het niveau dat je echt een gesprek kan voeren, zeg maar.”

\subsection{Tema 2: Ferskaat}

Oanslute by de soarchfreger

Goed oanslute by de soarchfreger wurdt by alle opliedingen as in wichtich spearpunt sjoen. Dat wol sizze dat dêr rekken holden wurdt mei de yndividuele situaasje fan de soarchfreger en dêr sa goed mooglik yn meigien of meitocht wurdt. Rekken hâlde mei de taal en kultuer fan de soarchfreger is dêr in ûnderdiel fan. "Uitgangspunt van zorg is dat je dicht aansluit bij de leefwereld van je cliënt en taal is een onderdeel van die leefwereld," seit P17, opliedingsdirekteur by Ferpleechkunde \& Fakterapy. Dat útgongspunt sit wol ymplisyt yn de fyzje fan opliedingen, mar net eksplisyt. Oanslute by de soarchfreger spilet in grutte rol yn it kurrikulum by fakken lykas Metodyk of Sosjale Feardichheidstrainingen en by it weromkeppeljen nei staa- 


\section{US WURK LXX (2021), p. 42}

zjes. Kultuer wurdt dêr soms besprutsen, mar taal hast noait eksplisyt. It Frysk komt dêrby ek net folle oan 'e oarder, behalve as in studint dêr sels in kasus oer oandraacht.

Yn it wurkfjild wurdt oanslute by de soarchfreger ek as in belangryk spearpunt sjoen. Faak waard dêrby neamd dat de soarchfreger syn of har memmetaal, bygelyks it Frysk, prate kinne moat en dat de wurknimmer dat ferstean kin (of in oare oplossing sykje moat). "Ik vind het heel belangrijk dat je als therapeut sowieso aansluit bij de cliënt die je in behandeling hebt en met name de taal is daarin ook een heel belangrijk punt," seit P11, terapeut by de GGZ. Ek P2, koördinator by Zienn, jout oan: "Hulpverlening gaat voor een heel groot gedeelte over vertrouwen en aansluiting en als je de taal spreekt, bijvoorbeeld Fries met mensen die echt native Fries praten, dan sta je wel met 1-0 voor, zeg maar, als je dat kunt aanbieden.”

\section{Kultuerferskillen}

Yn de opliedingen is der wol wat omtinken foar kultuerferskillen. Yn it wurkfjild wurdt oanjûn dat by de measte ynstellingen kultuerferskillen net sa faak foarkomme, en as dy der dochs binne en foar problemen soargje kinne, dat dy problemen meastal goed (ad hoc) oplost wurde kinne. Der wurdt wol oanjûn dat kultuerferskillen eins noch lestiger wêze kinne as taalferskillen. P6, ferpleechkundige MCL, fertelt bygelyks dat yn sommige kultueren net hiel iepen mei bern kommunisearre wurdt. Sa kaam hy derefter dat in bern op syn offdieling sels net wist dat it kanker hie. "Wel dat hij ernstig ziek is, en dat voelt hij zelf ook wel, maar dat vind ik zelf wel heel lastig."

Frysktaligen fs. net-Frysktaligen yn de opliedingen

By alle opliedingen, benammen Fakterapy, dêr't in soad Dútske studinten binne, wurdt oanjûn dat net alle studinten út Fryslân komme en/of yn Fryslân bliuwe sille te wurkjen. Dat wurdt keppele oan de relevânsje fan it Frysk: de studinten dy't aanst dochs wer fuortgeane, kinne der net folle mei en binne der ek net yn ynteressearre.

P18, teamlieder Fakterapy, seit:

"Wij hebben best wel veel studenten die niet uit Friesland komen. Die hebben daar niet zoveel mee [de Friese taal], die gaan gewoon weer terug naar waar ze vandaan komen." 


\section{US WURK LXX (2021), p. 43}

Der wurdt oanjûn dat de needsaak om eventueel Frysk te learen mar foar in hiele lytse groep fan harren studinten ynteressant wêze soe: dyjingen dy't noch gjin Frysk prate, mar wol yn Fryslân wurkje wolle.

Yn it wurkfjild wurdt oanjûn dat net-Frysktalige wurkimmers faak geandewei it Frysk leard ha te ferstean, en dat guon it ûnderwilens ek al prate kinne. "Mijn spreektaal Fries is begonnen toen ik in de zorg ging werken," jout P6, ferpleechkundige yn it MCL en opgroeid bûten Fryslân, oan.

\section{Bewustwêzen fan meartaligens}

Soarchferlieners binne har derfan bewust dat meartaligens in rol spilet yn harren sektor, dat wol sizze, alle dielnimers jouwe oan geregeld yn oanrekking te kommen mei meartaligen (faak Nederlânsk/Frysk) en wolle dan graach oanslute by de soarchfreger. In pear jouwe oan dat se dêr wol wat oer leare wold hiene yn de oplieding. "Er wordt eigenlijk geen aandacht aan besteed, terwijl het juist in de praktijk heel belangrijk is. Dus ik denk vooral dat bewustwording wel iets is om mee te beginnen ja," fynt P4, manager by Sionsberg.

Yn de oplieding spilet bewustwêzen fan meartaligens yndirekt mei by ferskillende fakken, as der omtinken is foar kommunikaasje, oansluten by de soarchfreger en kultuerferskillen, mar taal wurdt hast noait eksplisyt beneamd. As taal wol beneamd wurdt, giet it meastal net om it Frysk, mar earder om migrantetalen, lykas Arabysk. It feit dat Frysk ek in memmetaal is en dat soarchfregers it belangryk fine om dy te praten, sit net eksplisyt yn it kurrikulum. Neffens ferskate belutsenen by de oplieding is it wol belangryk om dat ta te foegjen. "Dat je mensen met verschillende achtergronden en verschillende talen hebt, het is goed om daar ook bij stil te staan, wat dat doet," seit P16, opliedingsdirekteur Social Work.

P18, teamlieder Fakterapy, jout oan dat sok bewustwêzen goed passe soe yn in fiktive kasus:

"In jaar 1 werken we met fictieve casussen, dan komen ze niet zozeer in aanraking met de praktijk of met echte cliënten. Daar [bewustzijn meertaligheid] zou je natuurlijk ook een mooie casus over kunnen schrijven."

Ek P23, dosint Social Work, komt mei in eigen oanrekommandaasje:

"Een soort vak als regiokunde, dus waarbij je aandacht hebt voor specifieke identiteit van een werkgebied waar je werkzaam bent, waar taal belangrijk in is, dat zou voor mij aanbeveling nummer 1 zijn." 


\section{US WURK LXX (2021), p. 44}

\subsection{Tema 3: Wearde memmetaal}

Yn it wurkfjild wurdt oanjûn dat it foar in soarchfreger o sa belangryk is dat er syn memmetaal prate kin. By de opliedingen binne de antwurden ek posityf as frege wurdt oft it belangryk is dat in soarchfreger syn of har memmetaal prate kin; it is yn 'e praktyk lykwols net altyd mooglik om dat te dwaan. Ut de ynterviews komt nei foaren dat it praten fan de memmetaal yn de soarch twa belangrike funksjes hat: it skept in bân en men kin gefoelens better uterje.

Bân skeppe

It praten fan de memmetaal kin de ôfstân tusken de soarchfreger en soarchferliener lytser meitsje, jouwe soarchferlieners oan. P3, unitlieder MCL seit:

"In de zorg als het over jezelf gaat en dichtbij jezelf komt, dan is het heel fijn dat je dan met elkaar dezelfde taal kan spreken. Je begrijpt elkaar dan goed, je voelt elkaar aan en het geeft een stuk vertrouwen."

By Veilig Thuis wurdt dat ek sjoen. P7, meiwurker Veilig Thuis, fertelt:

"De drempel om Veilig Thuis te bellen is al een hele hoge. Als je dan iemand spreekt waarbij je het gevoel hebt van hé, wij hebben iets gemeenschappelijks zeg maar, wij hebben een lijntje, dat kan soms, en zeker bij ouderen denk ik, echt wel een pré zijn."

\section{Gefoelens uterje}

Ferskillende soarchferlieners jouwe oan dat it uterjen fan gefoelens en emoasjes it bêste giet yn de memmetaal, en dat se soarchfregers dêrom graach dy romte biede.

"Ik denk dat iemand z'n gevoelens beter kan uiten wanneer je je eigen taal spreekt," seit P5, manager by GGZ.

P10, begelieder by Zienn, tinkt der ek sa oer:

"In hun moedertaal kunnen ze zich beter uiten," seit sy.

P11, terapeut by GGZ, jout oan dat se it ek weromkrijt fan soarchfregers:

"Ik hoor heel vaak van cliënten terug, hè Friestalige cliënten, die kunnen dat prima in het Nederlands, maar dat het toch vaak een stukje meer is, een stukje makkelijker is, een stukje gevoelsmatig dichter bij zichzelf komen, als ze het in het Fries kunnen doen." 


\section{US WURK LXX (2021), p. 45}

Yn de opliedingen wurdt dat op grûn fan praktykûnderfiningen ek wol ynsjoen, mar der wurdt fierder gjin eksplisyt omtinken oan jûn.

\subsection{Tema 4: Taalbelied}

Opfallend as it giet om it tema taalbelied is dat de measte ynstellingen oanjouwe gjin offisjeel taalbelied te hawwen, offsjoen fan it feit dat kommunikaasje nei de bûtenwrâld yn it Nederlânsk giet. De taalkar yn it kontakt mei soarchfregers giet faak "fansels", men fernimt gau genôch dat immen Frysktalich is en as wurknimmer en soarchferliener beide Frysk prate, wurdt dêr yn 1-op-1-situaasjes meastal op oerskeakele, om't dat nofliker is.

P10, begelieder by Zienn, seit dêroer dat der net eksplisyt nei taalfoarkar frege wurdt.

"Meestal hoor je het wel aan elkaar, dat je Fries spreekt. In principe spreken we hier Nederlands, natuurlijk, en als het anders is, dan schakelen we over."

As in soarchfreger in taal praat dy't de wurknimmer net ferstiet, hawwe de measte soarchynstellingen de mooglikheid fan in tolketelefoan, al wurdt faak besocht om it earst yntern op te lossen.

P3, unitlieder yn it MCL, seit:

"Het is soms lastig om daar [tolkentelefoon] een tolk te krijgen, en omdat het ook vrij kostbaar is, hebben we gewoon intern gekeken: goh, welke mensen hebben we? Wie spreekt wat? Zodat je het op die manier kan faciliteren en dat het vaak ook medisch onderlegde mensen zijn."

By guon bedriuwen wurdt by it oannimmen fan nije wurknimmers wol bepraat hokker talen oft de wurknimmer behearsket, mar binne der gjin kritearia foar taalfeardigens of is der gjin offisjeel belied foar fêststeld.

P5, manager by GGZ, seit:

"Ik vind het belangrijk [dat iemand Fries verstaat], maar ik weet dat een collega-manager dat niet vindt. Er is geen beleid op."

\section{Diskusje}

It doel fan dit ûndersyk wie om derefter te kommen hoe't yn Fryslân de hbû-opliedingen soarch en wolwêzen it bêste oanslute kinne by de soarchpraktyk op it mêd fan Frysk en meartaligens. Om dêrefter te kommen waard 


\section{US WURK LXX (2021), p. 46}

ûndersyk dien nei it ferlet yn it wurkfjild, nei it ferlet en stân fan saken by de opliedingen en hoe't dy twa it bêste op elkoar oanslute kinne.

Op grûn fan boppesteande resultaten (sjoch seksje 4. Resultaten) kinne we konkludearje dat der yn it wurkfjild ferlet is fan it brûken fan it Frysk en fan bewustwêzen fan meartaligens (ûndersyksfraach 1). Der wurdt op it stuit in soad gebrûk makke fan it Frysk, benammen yn ien-op-ien situaasjes. Soarchferlieners fine it belangryk dat wurknimmers Frysk ferstean kinne, om goed oan te sluten by de soarchfreger en omdat de measte minsken har it bêste uterje kinne yn de memmetaal. Dat slút oan by eardere befiningen yn Fryslân (Afûk, 2013) en dy fan ferlykber ûndersyk yn Wales (Wagoner, 2017). Dat soarchferlieners Frysk prate kinne, wurdt troch harren sels sjoen as in plus, mar it wurdt minder belangryk fûn as Frysk ferstean. Soarchferlieners rinne ek wol ris tsjin kultuerferskillen en kommunikaasjeproblemen oan by minsken mei in migraasje-eftergrûn. Dat kin goed oplost wurde; de soarchferlieners fine it belangryk dat der bewustwêzen is fan meartaligens (ek fan it Frysk) en de rol dy't meartaligens spilet by de soarchfregers.

Yn de opliedingen - in aspekt dat oant no ta minder oandacht yn de literatuer krigen hat - wurdt op dit stuit lykwols hast gjin omtinken jûn oan it Frysk (ûndersyksfraach 2). It Frysk liket net foar alle studinten relevant te wêzen, omdat in grut part óf net út Fryslân komt en dêr ek net bliuwe sil, óf it al praat. De dosinten en teamlieders sjogge dus net it nut yn fan in kursus Fryske taalfeardigens, mar sjogge wol de wearde dy't it praten fan de memmetaal troch soarchferlieners hat foar soarchfregers. Ek is der no mar in bytsje eksplisyt omtinken foar taal. Hoewol't der wol fokust wurdt op "goed oansluten by de kontekst fan de soarchfreger" en kultuerferskillen dêr in rol by spylje, mei der neffens de ûnderfrege belutsenen wol mear omtinken wêze foar taal yn dy persoanlike kontekst. Der binne gjin grutte ferskillen fûn tusken de opliedingen. Op grûn fan ús befiningen hawwe wy in oanrekommandaasje skreaun foar de opliedingen om meartaligens in natuerlik plak te jaan yn it kurrikulum, sûnder dat der eksplisyt oan taalfeardigens wurke wurdt (ûndersyksfraach 3).

Hoewol't ferskillende eardere ûndersiken de grutte wearde fan minderheidstalen yn de soarch oankaarte hawwe, sawol nasjonaal (cf. Loeven, 2005, Afûk, 2013 en Berends, 2020) as ynternasjonaal (cf. Schenker et al., 2008, Berufsbildende Schulen des Landkreises Oldenburg, 2015, Wagoner, 2017), en ek de fraach nei meartalich soarchpersoniel (Roberts en Paden, 2000, Afûk, 2013), is der gjin ûndersyk bekend nei soarch- en wolwêzensopliedingen, dy’t dochs it begjinpunt binne om minderheidstalen ûnder de 


\section{US WURK LXX (2021), p. 47}

oandacht te bringen by (takomstige) soarchferlieners. It ûndersyk dat wy dien hawwe is ynnovatyf yn dy sin, dat der net allinne yn it wurkfjild mar ek by de opliedingen sjoen is nei it plak fan minderheidstalen, en dat der in grutte fokus wie op de oansluting tusken opliedingen en wurkfjild.

It ûndersyk hat in pear tekoartkommings, benammen as it giet om de ynterpretaasje fan de ûndersyksresultaten. Dy binne it gefolch fan de stekproef dy't op basis fan teoretyske sampling en snowballsampling lutsen is. Dat leveret in beheind tal soarchynstellingen op, sadat der mear ferskillen yn it wurkfjild bestean kinne as dat no nei foaren kommen binne. In faktor dy't dêr in rol by spylje kin, is de spesifike doelgroep dêr't de soarchferliener mei wurket, en ek de (gemiddelde) leeftiid fan de doelgroep. Yn in ferfolchûndersyk kin útsocht wurde oft der ferskil is tusken âldere en jongere soarchfregers, as it giet om de fraach oft se it leafst in minderheidstaal brûke (en wat de ynstellingen dêrfan fine). Boppedat soe yn in ferfolchûndersyk neigien wurde moatte, oft der (grutte) regionale ferskillen spylje, om't it Frysk net oeral yn de provinsje likefolle praat wurdt; sa waard no yn it ûndersyk troch in meiwurker yn Dokkum oanjûn dat dêr folle mear Frysk praat wurdt as yn bygelyks Ljouwert en Harns. Dochs kin ús ûndersyk sjoen wurde as in fariearre ferkenning fan ferskillende kanten fan de soarch- en wolwêzenssektor. It biedt ús in soad ynsjoch yn it ferlet yn it wurkfjild en de stân fan saken en behoeften by de hbû-opliedingen.

Konkludearjend kinne we sizze, dat meartaligens en minderheidstalen in grutte rol spylje yn de soarch- en wolwêzenssektor, mar dat de soarch- en wolwêzensopliedingen op dit stuit noch net folle oandacht oan dy rol besteegje. "In principe spreken we hier Nederlands, natuurlijk,", it sitaat dêr't dit artikel mei begjint, is as útgongspunt yn de soarch- en wolwêzensektor miskien net hielendal korrekt. Want sa fanselssprekkend blykt it net te wêzen om allinne mar it Nederlânsk en net (ek) de regionale taal te brûken.

NHL Stenden Hogeschool

Ljouwert/Leeuwarden 


\section{US WURK LXX (2021), p. 48}

\section{REFERINSJES}

Anderson, L., Scrimshar, S., Fullilove, M., Fielding, J., \& Norman, J. (2003). Culturally competent healthcare systems: A systematic review. American Journal of Preventative Medicine 24, 68-79.

Afûk. (2013). Hokker taal prate jo? Yn: http://www.gemeentenenfrysk.nl/upload/files/8.\%20sunenssoarch/Hokker _taal_prate_jo_nl-fr.pdf

Berends, N. (2020). Met streektaal sneller een band. Nursing 26(3), 28-31.

Berufsbildende Schulen des Landkreises Oldenburg (2015). Plattdeutsch in der Pflege - Wi snackt Platt... du ok? (Ein kleiner Hoch-Platt-Übersetzer für den Pflegealltag, 2. Auflage).

Blömer, J., Boneschansker, E., Tuin, I. van der (2001). Taal in de zorg/Taal yn 'e soarch. Ljouwert: Economisch Bureau Coulon.

Braun, V. and Clarke, V. (2006). Using thematic analysis in psychology. Qualitative Research in Psychology 3 (2), 77-101.

Chen, A.H., Youdelman, M.K., \& Brooks, J. (2007). The Legal Framework for Language Access in Healthcare Settings. Journal of General Internal Medicine 22, 362-367.

Clark, M. M. (1983). Cultural context of medical practice. Western Journal of Medicine 139(6), 806.

Coffi, J. (2003). Communicating with culturally and linguistically diverse patients in an acute care setting: nurses' experiences. International Journal of Nursing Studies 40, 299-306.

Davies, E. (2009). They all speak English anyway. Cardiff: Care Council for Wales.

Hopf, C. (2004). Qualitative interviews: an overview. In: Flick, U., von Kardoff, E. \& Steinke, I. (Eds)., A companion to qualitative research. London: Sage publications, 203-208.

Institut für niederdeutsche Sprache e.V. Bremen (2016). Schnack doch maal Platt mit mi! - Bildungsurlaub für Pflegekräfte. http://www.ins-bremen.de/de/sprache/bildung/luechttorns.html

Irvine, F. E., Roberts, G. W., Jones, P., Spencer, L. H., Baker, C. R., \& Williams, C. (2006). Communicative sensitivity in the bilingual healthcare setting: A qualitative study of language awareness. Journal of Advanced Nursing 53(4), 422-434.

Johnson, M., Noble, C., Matthews, C., \& Aguilar, N. (1998). Towards culturally competent healthcare: language use of bilingual staff. Australian Health Review 21, 49-66. 


\section{US WURK LXX (2021), p. 49}

Krosenbrink, H. (1983). Volkswijsheden uit de Achterhoek. Twents-Gelderse Uitgeverij W.G. Witkam, Enschede.

Labov, W. (1972). Sociolinguistic patterns. University of Pennsylvania Press, Philadelphia.

Lavizzo-Mourey, R. (2007). Improving quality of US health care hinges on improving language services. Journal of General Internal Medicine 22, 279-280.

Loeven, J.M. (2005). Als je plat kunt praten, moet je het niet laten: Onderzoek naar de mogelijke meerwaarde van het gebruik van streektaal in de zorg (Master's thesis, University of Twente).

Mack, N., Woodsong, C., MacQueen, K., Guest, G., \& Namey, E. (2005). Qualitative research methods: A data collector's field guide. Research Triangle Park, NC: Family Health International.

Roberts, G.W., \& Paden, L. (2000). Identifying the factors influencing minority language use in health care education settings: a European perspective. Journal of Advance Nursing 32(1), 75-83.

Robinson, M. (2002). Communication and health in a multi-ethnic society. Bristol: Policy Press.

Schenker, Y., Lo, B., Ettinger, J.D., \& Fernandez, A. (2008). Navigating language barriers under difficult circumstances. Annals of Internal Medicine 149, 264-269.

Scharlmann, A. (2016). Niederdeutsch in der Pflege. Ms.Carl von Ossietzky Universität Oldenburg.

Wagoner, C. (2017). Language Capacity Building and Strengthening in the Welsh Statutory Education and Health and Social Sectors. PhD. Cardiff. 